\title{
Managing the water-energy-food nexus on an integrated geographical scale
}

Dr. Ammar Abulibdeh, Department of Humanities; Dr. Esmat Zaidan, Department of International Affairs

\section{Abstract}

The water-energy-food (WEF) nexus is the subject of much research focusing on different aspects, a wide range of issues, and development of a variety of models and tools. This study takes a different approach by developing a holistic framework that concentrates on the spatial elements of continuity and change associated with WEF transition on national, regional, and international scale. The study also investigates the interconnected challenges that could affect these resources and the actions and polices that should be taken on different geographical scales to address these challenges. The results can help practitioners and policy makers gain a clearer understanding of the state of the knowledge when performing WEF nexus assessments at different geographical scales.

\section{Introduction}

The main aim of the WEF nexus is to transmit normative implications that consider the processes of producing, distributing, and consuming WEF resources into a decisionmaking process and to manage them more effectively and efficiently. The significance of the WEF nexus is manifest, because of the increased stress and shortages of these resources, accompanied by rapid population growth and economic prosperity, changing consumption patterns, climate change, and competing landuse patterns. Therefore, it is considered a major component in national sustainable development strategies and simultaneously as an indicator of the level at which WEF security objectives are achieved (Golam and Sharma, 2015; Biggs et al., 2015; Endoa et al., 2017).

\section{Purpose of the study}

The overall aim of this study was to develop a holistic and comprehensive systemic framework to optimize WEF resources management and to capture the integration and interactions between these resources, in order to contribute to economic development at the national, regional, and international levels. A further aim was to investigate the interconnected challenges that could affect these resources, and the actions and polices that should be taken on different geographical scales to address these challenges. The interlinks between national, regional, and international economies, sector policies, and cross-sector impacts are not internalized into national policies. The intention with the framework developed in this study was to investigate geographical integration of WEF sectors through the nexus and the integration of policy options for minimizing trade-offs and maximizing synergies regarding these resources. Another intention was to develop a holistic approach for better coordination of these resources at different geographical scales.

The study focused on the Gulf region, an arid and semi-arid region characterized by water and food scarcity, but rich in energy resources. The WEF nexus in this region can be explored at multi-geographical scales. Therefore, the work focused on framing WEF based on integrated geographical scales (i.e., national, regional, and international levels).

\section{The geographical framework of the WEF} nexus

Framing the geographical interconnectedness of WEF sectors is crucial for understanding the coherence and strength of coordination between these sectors. It helps decision makers develop a holistic view of the linkages between WEF sectors and shifts the decision-making process towards developing institutional mechanisms to synchronize the decisions of different authorities and to strengthen synergies and complementarities among the three resources at various geographical scales.

Figure 2 shows the structure of our holistic geographical framework for WEF resources. A bottom-up approach was used to develop the framework, encompassing the linkage between WEF resources at different geographical scales. The framework captures the national, regional, and international level dimensions of the WEF nexus and has different dimensions that can assist appropriate policy formation and analyze the interdependencies of these resources at different geographical scales. It involves two steps:

1) Specifying the interconnectedness and integration between these resources on national, regional, and international geographical scales. This can help find a suitable strategy to achieve sustainable and efficient management of these resources.

2) Evaluating the risks threatening the interconnectedness of these resources at different geographical scales. This can help in planning for new strategies to overcome these risks.

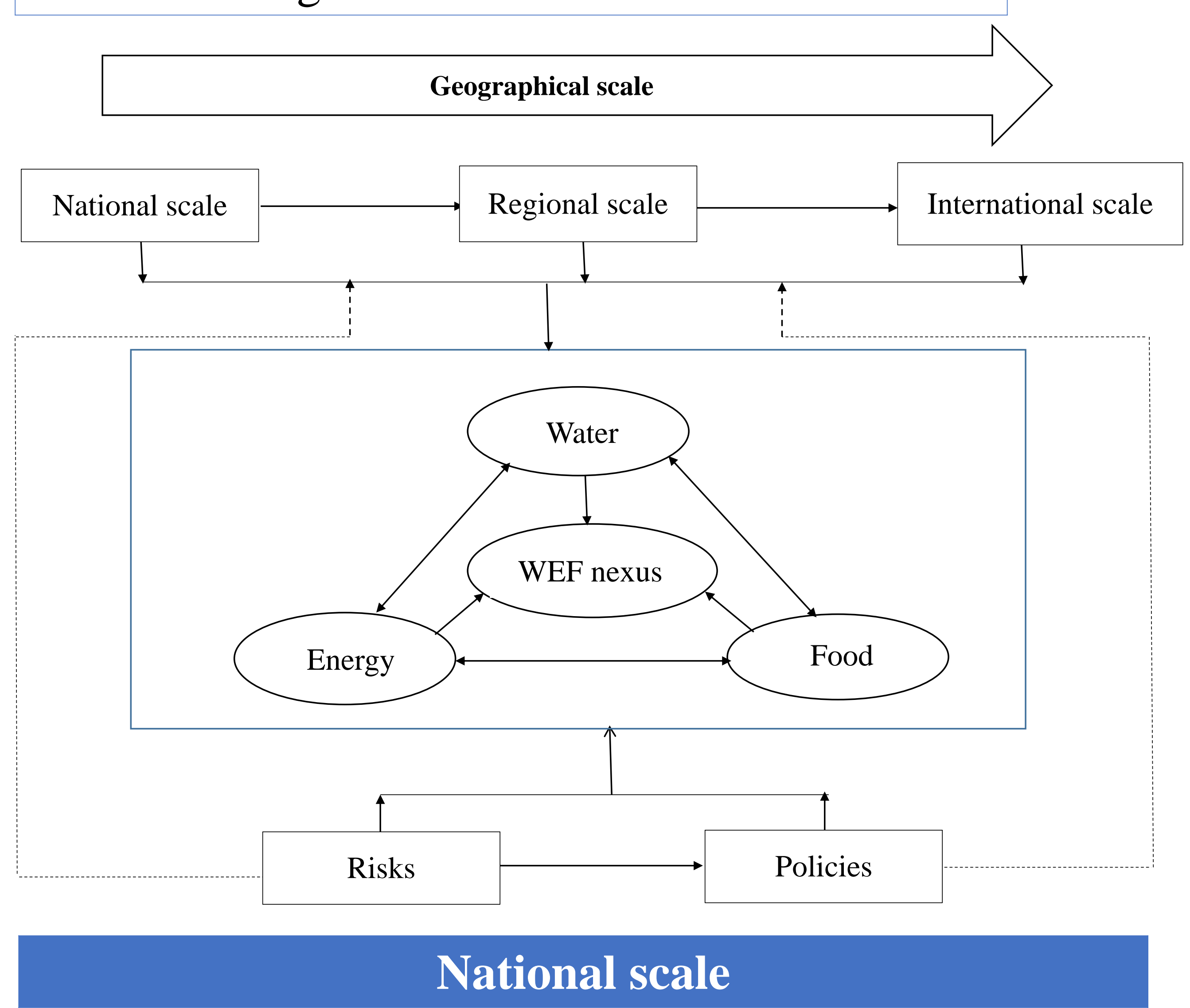

At the national scale, the key variables of the WEF nexus are at the household, rural area, urban area (city), and national levels, as shown in the national-scale framework (Figure 3). The framework addresses the interaction between WEF sectors starting from the enduse level at the household scale, then moving up to investigate the nexus at other geographical sub-scales within the national scale.

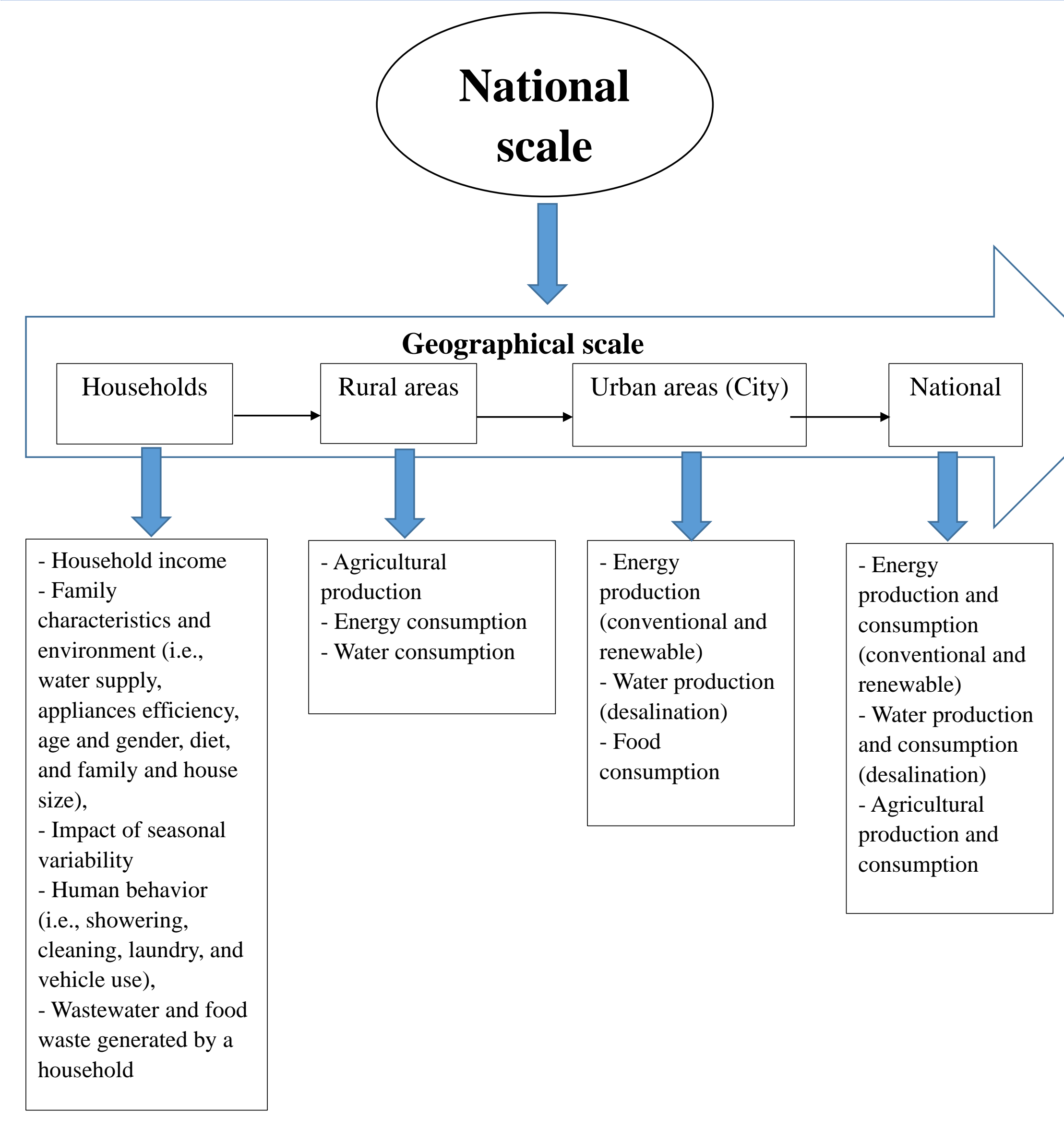

\section{Regional scale}

Integration and collaboration between the countries in a region is significant in addressing some of the important challenging aspects of the WEF nexus. The countries in the Gulf region have similar characteristics regarding the WEF resources, i.e., they are rich in conventional energy resources but scarce in water and food resources, and have water and food security challenges at the national level.

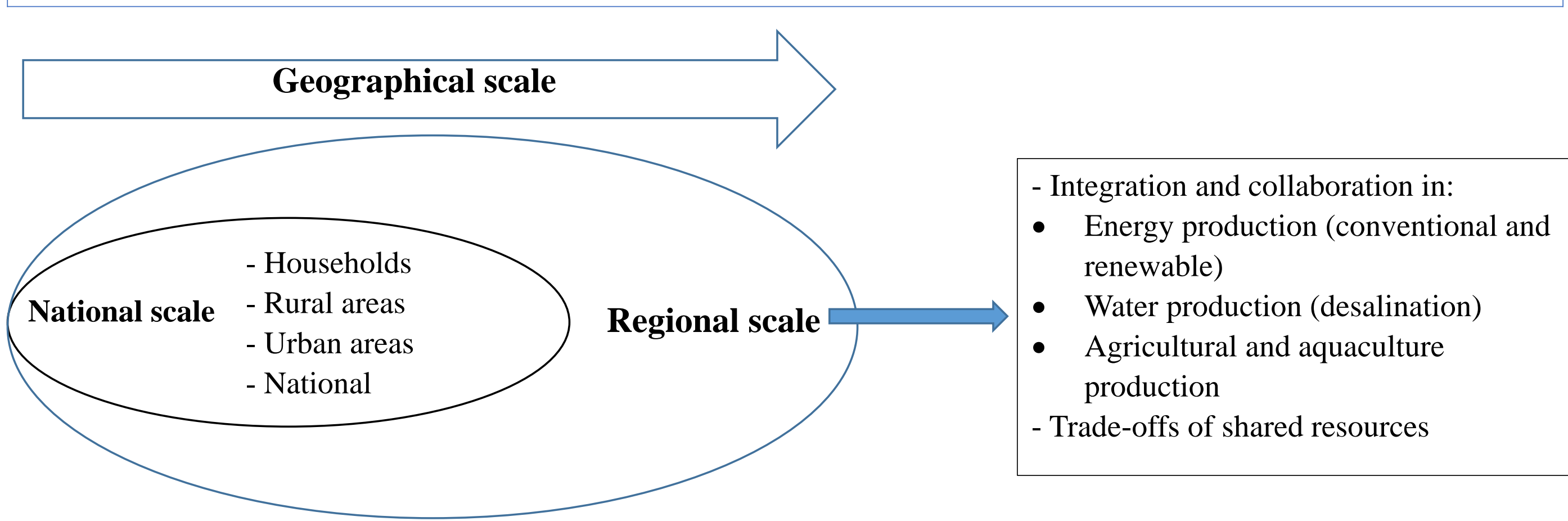
International scale

The benefits of development and progress in WEF security are very unequally distributed between and within countries across the globe. The level of global WEF security is under pressure from national, regional, and international changes, such as economic and population growth and changing lifestyles. On the international scale, WEF security can be achieved through a nexus approach involving integrated management and governance of WEF issues.

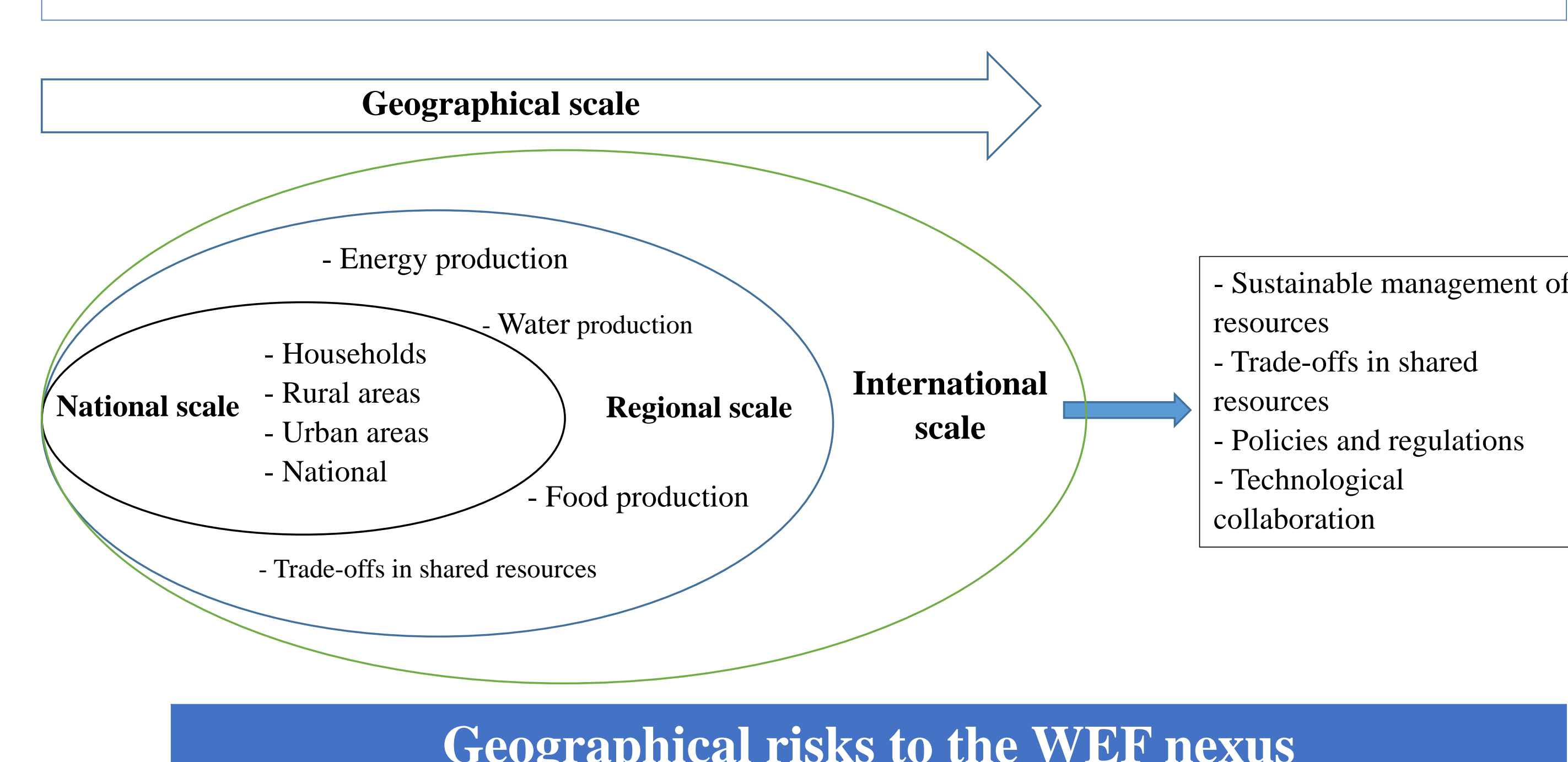

Generally, risks to the WEF nexus are associated with certain events, which can have a detrimental effect on security, economic, environmental, and technological issues. These risks have impacts on WEF resources and security at different geographical levels.

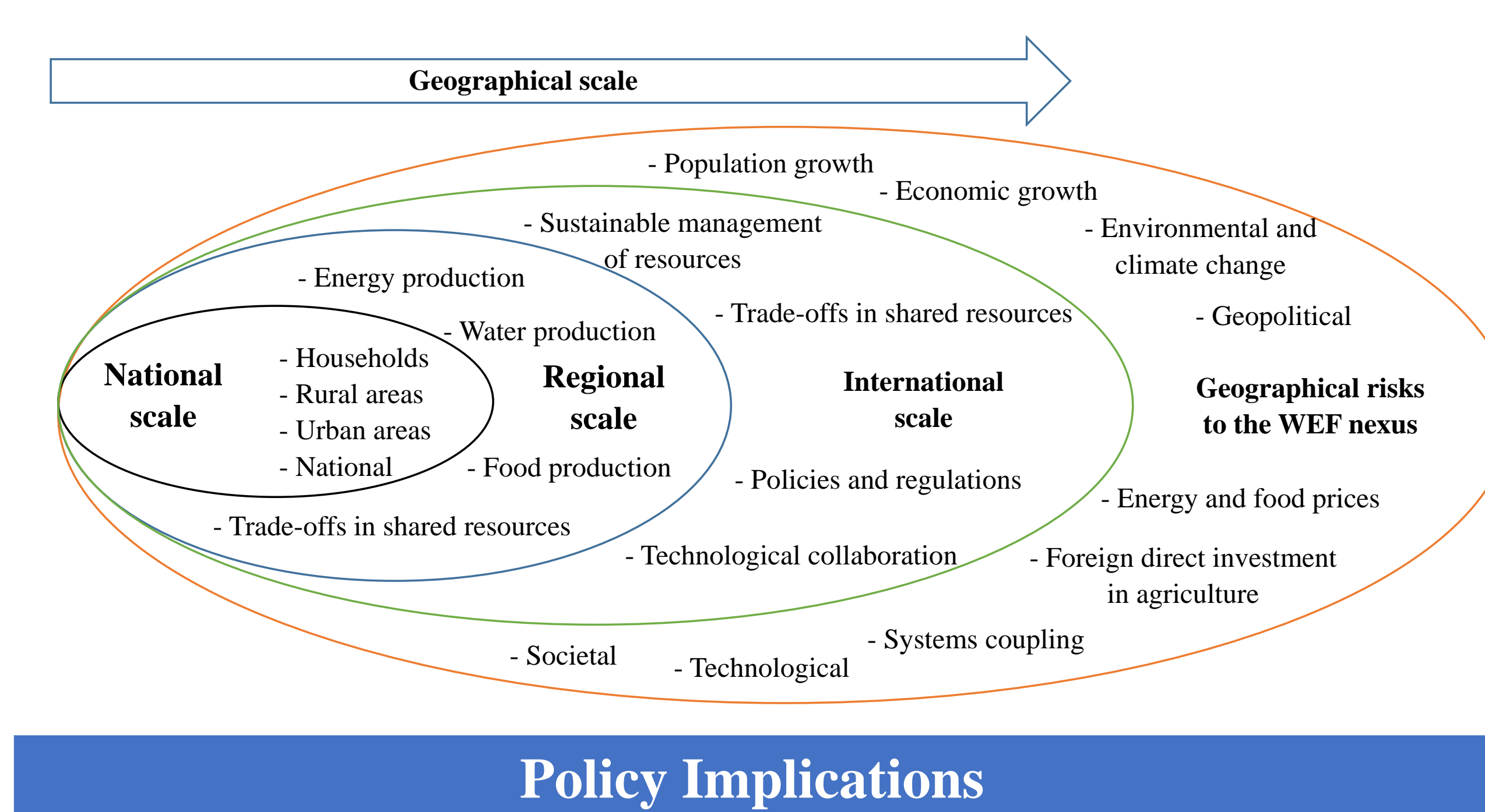

The process of achieving geographical collaboration and integration is not limited to the WEF resources themselves, but extends to the development polices and implications for resources security at different geographical scales.

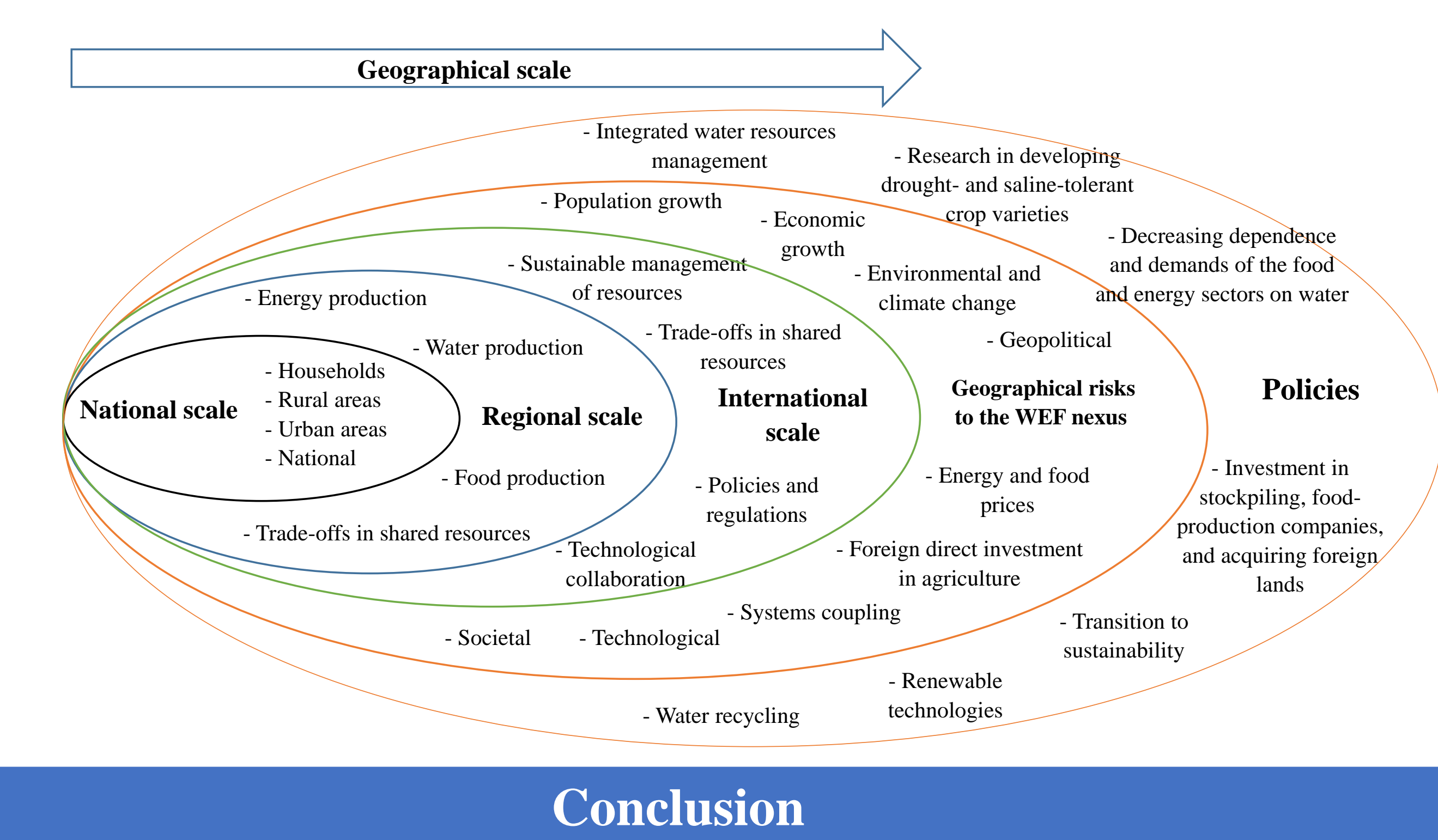

The holistic geographical framework developed in this study addresses the significance of the WEF nexus at the national scale and the importance of integration and collaboration between countries at the regional and international scales to achieve WEF security. The WEF nexus approach is important in the transition to sustainability, by generating benefits that outweigh the transaction costs and reducing trade-offs associated with stronger integration across sectors. Achieving WEF security cannot be attained by each country in isolation from its surrounding neighbors or from other countries across the globe. On the national level, it is a duel responsibility for residents and authorities. Policies that decouple economic and population growth from resource use and enable transition to a more circular economy are essential in order to achieve the sustainability targets in the national visions of the GCC countries. 